\title{
An upper- and lowercase alphabetic similarity matrix, with derived generation similarity values
}

\author{
DAVID B. BOLES and JOHN E. CLIFFORD \\ Rensselaer Polytechnic Institute, Troy, New York
}

\begin{abstract}
A full upper-and lowercase visual similarity matrix is presented for a standard set of computer characters, implemented on the Apple-Psych system. The 2,704 $(52 \times 52)$ letter pairs were rated by 12 subjects each. From the ratings, generation and veridical similarity values are derived, and they are tabled for use in research on mixed-case letter matching. In addition, the results of multidimensional scaling and cluster analyses are presented, which give complementary, simplified descriptions of the data.
\end{abstract}

The impact of visual similarity on pure-case letter comparisons (e.g., between " $A$ " and " $A$ ") is well documented. Letters that are commonly confused with one another (e.g., "GQ") produce longer reaction times (RTs) in same-different matching tasks than do letters that are rarely confused (Bagnara, Boles, Simion, \& Umiltà, 1983). This effect is a source of evidence that pure-case letter pairs are matched physically (visually), and, indeed, the task of matching such pairs is known as the physical matching task (Posner, 1978).

Mixed-case letter comparisons (e.g., between " $A$ " and " "a") have come to be known as name matches, because introspection and early experimental results suggested that such matches are made by extracting and comparing phonetic representations of the letter names (Dainoff \& Haber, 1970; Posner, Boies, Eichelman, \& Taylor, 1969; Thorson, Hochhaus, \& Stanners, 1976). However, more recent work indicates that phonetic or name codes do not typically underlie such matches (Besner, Coltheart, \& Davelaar, 1984; Boles, 1986; Boles \& Eveland, 1983). Thus, Boles and Eveland (1983) showed that phonetically similar different pairs (e.g., "Gd") produce no increment in RT, contrary to what is predicted if name codes are used. In addition, mixed-case matches fail to produce a right-visual-field superiority with lateralized presentation, again contrary to prediction if left hemisphere phonetic processes are involved. In contrast, letter pairs judged same-different on the basis of rhyme do show such a superiority (Boles, 1986). The implication is that rhyme matches draw on phonetic processes, which are lateralized to the left hemisphere, while name matches do not.

In further investigations, Boles and Eveland (1983) and Boles (1986) formulated and tested a visual generation model of mixed-case matching. According to the model,

\footnotetext{
We would like to thank Michael E. Walker and Angela Ebreo for statistical consulting and assistance, and Susan Savage for entering data for the analyses. We would also like to thank Lester Krueger for comments on an earlier draft. Reprint requests should be sent to David B. Boles, Department of Psychology, Rensselaer Polytechnic Institute, Troy, NY 12180.
}

one or both members of a presented mixed-case pair rapidly generate a visual representation of the opposite case in memory. For example, if both members generate the opposite case, the pair "Aa" might result in the generation of " $a A$ " in memory. The generated letter or letters are then compared to one or both of the original letters, supporting a type of visual match.

Evidence for the generation model was produced by manipulations of generation similarity. That is, different pairs were constructed in which the generated letters were visually similar to the original letters (e.g., "Gq" might generate "gQ"). RTs to such generation similar pairs proved to be substantially longer than to dissimilar pairs. The effect could not be attributed to visual similarity between the original letters themselves (called veridical similarity).

Unfortunately, a serious impediment to further pursuit of this work has been the absence from the literature of a visual similarity matrix using all crossings of both upperand lowercases of a standard font. This is an impediment because (1) generation similarity as used by Boles and Eveland (1983) and Boles (1986) is a derived index based on the similarities of the upper- and lowercase forms of a letter pair, and (2) in constructing generation similar and dissimilar letter sets for comparison, veridical similarity (e.g., between "G" and "q" for the pair "Gq") should be matched between the sets if this variable is to be controlled. Thus, a similarity matrix is needed that tables similarity values for both pure-case and mixed-case pairs. Existing matrices are generally based on confusions made in recognizing single letters, and so, in effect, they represent pure-case similarities (e.g., Gilmore, Hersh, Caramazza, \& Griffin, 1979; Townsend, 1971; van der Heijden, Malhas, \& van den Roovart, 1984).

In the absence of a matrix using both pure-case and mixed-case pairs, the experimenter must collect ratings for the particular font in use and compute the generation and veridical similarity indexes. The process is very timeconsuming, and, for practical reasons, it may require limitation to a small set of candidate letter pairs. Each candidate pair requires the rating of three or four actual 
pairs (for the candidate pair "Gq," the pairs "Gq," "GQ," "gq," and, if completeness is desired, "gQ"). Thus, Boles and Eveland (1983) restricted ratings to only 50 candidate pairs.

To overcome this methodological limitation, we now report a full similarity matrix using the upper- and lowercase versions of a standard set of characters. The characters are those used in the Apple-Psych system of experimental software, which is a system freely available and widely distributed in the public domain for Apple IIseries computers. It also has received recent description in a number of articles (Barnes \& Burke, 1988; Boles, 1988; Osgood, 1988). Thus, the matrix can be used by anyone implementing the Apple-Psych system and desiring to conduct letter-confusion research. To add to the utility of the results, we also report derived generation similarity values, so that these need not be computed. ${ }^{1}$

We must emphasize that our main purpose in constructing and reporting the matrix is methodological. The comparison of such models of visual confusion as template overlap, featural, spatial frequency, or choice models (Gervais, Harvey, \& Roberts, 1984; Loomis, 1982), while important, is beyond the scope of this paper. Nevertheless, we will report the outcomes of multidimensional scaling (MDS) and cluster analyses of the similarity ratings. This is to provide a description of the data that is simplified from the $2,704(52 \times 52)$ original letter pairs, and to point out structural aspects of the data that those taking a theoretical approach to letter similarity may want to consider.

\section{METHOD}

\section{Subjects}

Thirty-two undergraduate volunteers participated, receiving extra course credit. All had normal or corrected vision.

\section{Apparatus and Stimuli}

Letter pairs were generated and responses collected, using Apple-Psych software on an Apple IIe microcomputer. Letters were displayed in Apple-Psych size " 1 " on an Amdek 300 monitor with P31 phosphor, in highresolution mode. All 2,704 ordered pairs of upper- and lowercase letters (a $52 \times 52$ matrix) were used. The letter set is illustrated in Figure 1. Letters were constructed of illuminated pixels, generally in a $6 \times 7$ (horizontal $\times$ vertical) matrix. Letters with descenders (e.g., " $y$ ") involved an 8th row. The letter set is standard in the Apple-Psych system and is identical to the SYSTEM.CHARSET provided with Apple Pascal. Size varied somewhat with the letter, but to take a representative pair like " $\mathrm{Zm}$," the capital letter subtended $0.4^{\circ}$ both horizontally and vertically, and the lowercase letter $0.4^{\circ} \times 0.3^{\circ}$. Letters within a pair were immediately adjacent, with the gap between them amounting to about 1.4 '. The subjects used a chinrest to maintain a fixed viewing distance of $0.48 \mathrm{~m}$.

\section{Procedure}

The full matrix of pairs was randomly divided into eight subsets of 338 pairs each. Each subject was given three

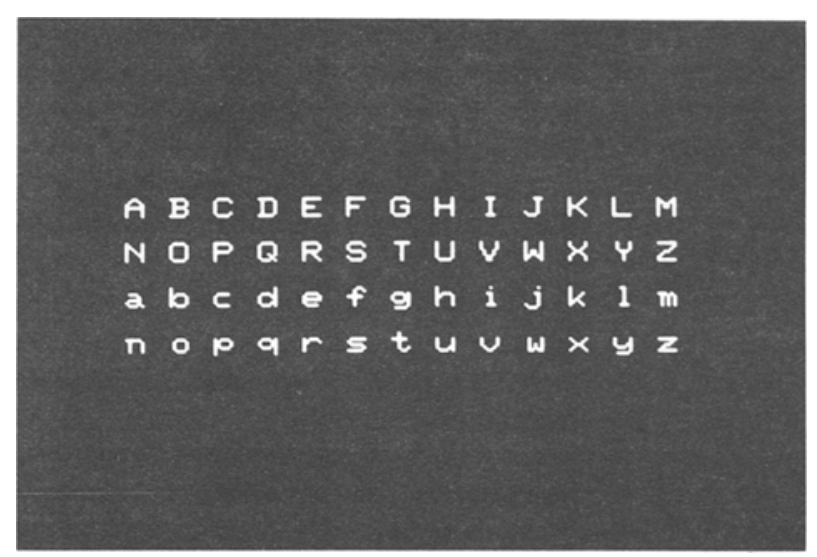

Figure 1. The Apple-Psych high-resolution letter set.

subsets to rate, with the subsets randomly selected and presented in random order, under the constraint that each subset was rated by 12 subjects. The pairs within a subset were also presented in random order.

On a given rating trial, the subject saw the heading "LOW $=1 \quad \mathrm{HIGH}=5$ " at the top of the screen, and a letter pair centered toward the bottom of the screen. The subject then pressed one of five keys, labeled 1-5, on the computer console, to indicate the rated similarity. They were instructed to rate the pairs on overall visual similarity, with " 1 " representing low similarity and " 5 " high similarity. The letters remained on the screen until the subject responded.

\section{RESULTS AND DISCUSSION}

Mean similarity values were calculated for each pair over subjects, with each pair having been rated by 12 subjects. These values are presented in Appendix A. Analysis indicates a high correlation between the two possible orders of the pairs, $r=+.88$, indicating that the matrix is substantially, though not perfectly, symmetric.

Summary data are shown in Table 1 , indicating the number of pairs falling into each of eight similarity bands, along with the mean (across pairs) of the standard devia-

Table 1

Summary Data of the Pairs

Classified by Pair Type and Similarity Band

\begin{tabular}{|c|c|c|c|c|c|}
\hline \multirow[b]{3}{*}{ Band } & \multicolumn{3}{|c|}{ Pair Type } & & \\
\hline & \multirow{2}{*}{$\frac{\text { Mixed }}{N}$} & \multirow{2}{*}{$\frac{\text { Upper }}{N}$} & \multirow{2}{*}{$\frac{\text { Lower }}{N}$} & \multicolumn{2}{|c|}{ All Pairs } \\
\hline & & & & $N$ & $S D$ \\
\hline $1.00-1.50$ & 608 & 7 & 48 & 663 & .61 \\
\hline $1.51-2.00$ & 530 & 198 & 256 & 984 & .87 \\
\hline $2.01-2.50$ & 128 & 279 & 188 & 595 & .99 \\
\hline $2.51-3.00$ & 44 & 109 & 83 & 236 & 1.03 \\
\hline $3.01-3.50$ & 16 & 46 & 51 & 113 & .99 \\
\hline $3.51-4.00$ & 11 & 10 & 20 & 41 & .88 \\
\hline $4.01-4.50$ & 14 & 1 & 4 & 19 & .75 \\
\hline $4.51-5.00$ & 1 & 26 & 26 & 53 & .19 \\
\hline
\end{tabular}

Note $-N=$ number of pairs in band. $S D=$ mean standard deviation. 
tions (across subjects) for the pairs within each band. Most pairs were rated fairly low in similarity, the mode being in the 1.51-2.00 band. Nearly all of the pairs in the highest band, of course, were physically identical pairs (e.g., "AA"). It is to be noted that not all of the identical pairs produced perfect similarity values of 5.00 , undoubtedly due to occasional keying errors by the subjects. Not surprisingly, the mean standard deviation is closely related to the scalar position of the band: Standard deviations were lower for the extremes (.61 and .19) than for the middle bands (1.03 and .99).

From these veridical similarity values, generation similarity can be computed. First, however, there is a complexity in the process that must be considered. Notably, the generation model makes rather unspecific claims as to what is generated and compared. The presented pair "Ab," for example, might generate only " $a$ " (which is then compared to " $b$ "), or only " $B$ " (compared to " $A$ "), or both " $a$ " and " $B$ " (respectively compared to " $b$ " and " $A$ "). Obviously, which model is adopted has a bearing on how generation similarity should be calculated. However, Boles and Eveland (1983) avoided the issue altogether by using as stimuli both forms of an original source pair (e.g., " $A b$ " and " $a B$ ") and calculating generation similarity in a joint manner (e.g., as the mean similarity of " $A$ " to " $B$ " and " $a$ " to " $b$ "). Regardless of the form of the model that is adopted, the joint generation similarity value describes the average generation similarity of the two forms of the source pair.

In short, we have followed this practice in computing the present generation similarity values. We have also collapsed over the two letter orders (e.g. , "Ab" and " $b A$ ") since, as noted above, the matrix is substantially symmetric. To take an example, the generation similarity of the pair " $A b$ " was calculated as the mean similarity of " $A B$ " (2.25), "ab" (2.66), "BA" (2.42), and "ba" (2.25), or a value of 2.40. The result of the computations is Appendix B, giving the generation similarity for each pair (upper entry) and, for convenience in constructing stimulus sets, the veridical similarity as well (lower entry). The tabled veridical similarity values were calculated under the same assumptions - that is, that both forms of a pair would be presented and that order is largely irrelevant. For example, the veridical similarity of "Ab" (1.52) reflects the mean similarity of " $A b$ " (1.42), " $a B$ " (1.75), "bA" (1.50), and "Ba" (1.42).

It is instructive to compare the present generation and veridical similarity values to those reported by Boles and Eveland (1983) for 50 letter pairs. In spite of the fact that somewhat different computer fonts were used in the two instances, the correlation between generation similarity values is $r=+.84$, and that between veridical similarity values is $r=+.74$. Evidently there is substantial similarity between the two fonts.

To better understand the subjects' ratings of similarity between letters, the similarity matrix in Appendix A (symmetrized by arithmetic averaging) was analyzed, using the nonmetric multidimensional scaling (MDS) program KYST-2A (Kruskal, Young, \& Seery, 1977). This pro- gram uses Torgerson's iterative procedure to fit Kruskal's (1964a, 1964b) model. An examination of the stress plot for the 2-through 5-dimensional solutions indicated the adequacy of fit of three dimensions. The decision to accept three dimensions was based on the form of the skree plot, with a pronounced elbow existing at three dimensions. In terms of a reduced number of dimensions, ours is a more conservative decision than that made by Gilmore et al. (1979), who accepted five dimensions on the basis of a low stress value (.077). Our stress value at three

Table 2

3-Dimensional Coordinates from the MDS Solution

\begin{tabular}{|c|c|c|c|}
\hline \multirow[b]{2}{*}{ Letter } & \multicolumn{3}{|c|}{ Dimension } \\
\hline & 1 & 2 & 3 \\
\hline A & -.89 & -.10 & -.28 \\
\hline B & -.64 & -.77 & .15 \\
\hline $\mathrm{C}$ & -.33 & -.62 & -.33 \\
\hline D & -.51 & -.82 & .00 \\
\hline$E$ & -.78 & -.32 & -.17 \\
\hline F & -.84 & -.48 & .41 \\
\hline G & -.35 & -.90 & -.32 \\
\hline H & -.88 & .26 & .07 \\
\hline I & -.81 & .08 & .74 \\
\hline $\mathbf{J}$ & -.42 & -.16 & .71 \\
\hline K & -.85 & .54 & .26 \\
\hline $\mathrm{L}$ & -.89 & .05 & .39 \\
\hline $\mathbf{M}$ & -.72 & .45 & -.61 \\
\hline $\mathrm{N}$ & -.74 & .44 & -.37 \\
\hline 0 & -.12 & -.78 & -.34 \\
\hline$P$ & -.25 & -.64 & .29 \\
\hline $\mathrm{Q}$ & -.32 & -.86 & -.53 \\
\hline $\mathrm{R}$ & -.76 & -.47 & .08 \\
\hline$S$ & -.32 & -.53 & -.90 \\
\hline $\mathrm{T}$ & -.72 & -.10 & .72 \\
\hline $\mathrm{U}$ & -.39 & .09 & -.32 \\
\hline $\mathrm{V}$ & -.37 & .77 & -.38 \\
\hline W & -.54 & .68 & -.56 \\
\hline$X$ & -.71 & .85 & -.25 \\
\hline $\mathrm{Y}$ & -.46 & .74 & .17 \\
\hline $\mathrm{Z}$ & -.75 & -.12 & -.85 \\
\hline $\mathbf{a}$ & 1.12 & -.29 & -.38 \\
\hline b & .54 & -.66 & .25 \\
\hline c & .58 & -.22 & -.34 \\
\hline$d$ & .66 & -.49 & .48 \\
\hline e & .78 & -.47 & -.50 \\
\hline f & .29 & -.27 & 1.06 \\
\hline g & 1.08 & -.36 & .28 \\
\hline $\mathrm{h}$ & .42 & .30 & .34 \\
\hline $\mathrm{i}$ & .46 & .56 & 1.05 \\
\hline $\mathrm{j}$ & .66 & .16 & .80 \\
\hline k & .03 & .97 & .48 \\
\hline 1 & -.21 & .19 & 1.08 \\
\hline $\mathrm{m}$ & .82 & .63 & -.48 \\
\hline n & .79 & .31 & -.36 \\
\hline 0 & .77 & -.65 & -.28 \\
\hline$p$ & .88 & -.48 & .17 \\
\hline$q$ & .90 & -.68 & .26 \\
\hline $\mathrm{r}$ & .80 & .10 & .12 \\
\hline s & .76 & -.10 & -.86 \\
\hline$t$ & .38 & .16 & 1.00 \\
\hline $\mathrm{u}$ & .52 & .32 & -.26 \\
\hline$v$ & .39 & .81 & -.14 \\
\hline w & .35 & .80 & -.70 \\
\hline$x$ & .41 & 1.21 & -.07 \\
\hline$y$ & .71 & .56 & .13 \\
\hline$z$ & .49 & .32 & -.92 \\
\hline
\end{tabular}


dimensions was considerably higher (.178). Accordingly, the dimensional difference between the studies is more apparent than real, since if we had ignored the skree plot and selected a lower stress value, the solution would have included more dimensions. In any case, Table 2 gives the coordinates for the 3-dimensional solution.

Clearly, Dimension 1 is closely related to the upperversus lowercase status of the letters. All of the 26 uppercase letters show negative values on the dimension, while 25 of the 26 lowercase letters show positive values. The exception was "l," which some subjects may in fact have interpreted as "I.",

Dimension 2 also appears interpretable. It yields strongly negative values for a number of curved letters (e.g., B, D, G, O, Q) and strongly positive values for a number of letters containing straight slanted lines (e.g., $\mathrm{V}, \mathrm{X}, \mathrm{Y}, \mathbf{k}, \mathrm{v}, \mathrm{w}, \mathrm{x})$. While some slanted letters produced negative values (e.g., A, Z), their magnitudes were small. Letters composed only of horizontal and vertical lines, neither curved nor slanted, produced intermediate values. Thus, it seems appropriate to label Dimension 2 as curved versus slanted.

Dimension 3 appears to capture acute angular versus vertical letters. The former are composed of acute angles, and they tend to have negative values on the dimension (e.g., S, Z, s, w, z). Vertical letters have a predominantly vertical component and show positive values (e.g., I, J, $T, f, i, 1, t)$.

The symmetrized ratings were also subjected to three hierarchical clustering methods: complete linkage (Johnson, 1967), and the weighted (WPGMA) and unweighted (UPGMA) pair-group methods using arithmetic averages (Sokal \& Michener, 1958). Of these, WPGMA gave the most satisfactory results in terms of interpretability. The dendogram for the results of this analysis is given in Figure 2.

One can differentiate the clusters at either a proximal or a distal level, but general groupings can be described by examining those clusters emerging at the " 20 " level or higher. From left to right on Figure 2, the first such grouping $(Z, z, N, S, s)$ captures a number of the letters composed of acute angles, while the second $(E, F, L)$ contains letters showing both strong horizontal and vertical components. The third group (B, D, C, c, O, o, Q, G) contains mostly uppercase curved letters, along with their lowercase visual analogues, and the fourth $(\mathrm{K}, \mathrm{k}, \mathrm{X}, \mathrm{x}, \mathrm{H})$ contains vertically bisected letters that are symmetrical or nearly so about a horizontal axis. The fifth group contains largely diagonal letters $(W, w, M, V, v, U, u, y$, $Y, A)$. The sixth has letters with a strong vertical component ( $f, t, J, j, I, 1, i, T$ ), and the seventh has small humped letters $(n, h, r)$. The eighth and last group contains mostly curved lowercase letters (a, e, g, q, d, b, $P, p, R)$. The difference between the third and eighth groups may be that in the third, lowercase letters have clustered with their look-alike uppercase analogues $(\mathrm{C}, \mathrm{c}$, $\mathrm{O}, \mathrm{o}$ ), whereas in the eighth, the lowercase letters are mostly nonanalogues. The exceptions, " $p$ " and the physically similar letters " $P$ " and " $R$," may have clustered with the rest of the eighth group because of the similarity of "p" with " $q$," " $b$," and "d."

Comparing the outcomes of the MDS and cluster analyses, it is apparent that they were sensitive to somewhat differing aspects of letter similarity. The MDS analysis was considerably more sensitive to the case (upper vs. lower) of the letters. The cluster analysis was more sen-



Figure 2. The WPGMA cluster analysis. 
sitive to featural similarities. Specifically, clustering revealed eight groupings based on various combinations of acute angles, horizontal, diagonal and vertical lines, curves, and bisections, with some influence of case. The MDS analysis produced case as a nearly pure dimension, but other dimensions were sensitive to a reduced number of features, including acute angles, diagonal and vertical lines, and curves.

The differential outcome is interesting, because instead of considering dimensional and clustering analyses as complementary in nature, attention has often been directed toward considering one type of analysis as more appropriate than the other. Indeed, Pruzansky, Tversky, and Carroll (1982) provided statistical procedures for classifying a data set as favoring "spatial" (e.g., MDS) or "tree" (e.g., clustering) analyses. In contrast, our results suggest that useful information can be gained from combining approaches rather than selecting one over the other. In this, our conclusion differs from those of Gilmore et al. (1979) and Townsend (1971), both of whom concluded that a MDS solution yielded dimensions that were not readily interpretable. Yet even a cursory glance at Dimension 1 in Table 2 demonstrates ready interpretability. It is of course precisely on the methodological variable of upper-versus lowercase that our study differs from the previous ones.

In conclusion, the structural aspects of Table 2 and Figure 2 may be of interest to theorists wanting to model letter-similarity judgments. On the other hand, researchers interested in the effects of visual similarity on samedifferent judgments should find the data in Appendixes $A$ and $B$ to be of greatest interest.

\section{REFERENCES}

Bagnara, S., Boles, D. B., Simion, F., \& Umilta, C. (1983). Symmetry and similarity effects in the comparison of visual patterns. Perception \& Psychophysics, 34, 578-584.

Barnes, S., \& Burke, R. S. (1988). What is Apple-Psych? Behavior Research Methods, Instruments, \& Computers, 20, 150-154.

Besner, D., Coltheart, M., \& Davelaar, E. (1984). Basic processes in reading: Computation of abstract letter identities. Canadian Journal of Psychology, 38, 126-134.

Boles, D. B. (1986). Confusion and visual field effects in thyme and name letter matching. Perception \& Psychophysics, 39, 123-128.
BoLES, D. B. (1988). Voice recognition with the Apple-Psych system. Behavior Research Methods, Instruments, \& Computers, 20, 158-163.

Boles, D. B., \& Eveland, D. C. (1983). Visual and phonetic codes, and the process of generation in letter matching. Joumal of Experimental Psychology: Human Perception \& Performance, 9, 657-674.

DainofF, M. J., HABER, R. N. (1970). Effect of acoustic confusability on levels of information processing. Canadian Journal of Psychology, 24, 98-108.

Gervais, M. J., Harvey, L. O., Roberts, J. O. (1984). Identification confusions among letters of the alphabet. Journal of Experimental Psychology: Human Perception \& Performance, 10, 655-666.

Gilmore, G. C., Hersh, H., Caramazza, A., Griffin, J. (1979). Multidimensional letter similarity derived from recognition errors. Perception \& Psychophysics, 25, 425-431.

Johnson, S. C. (1967). Hierarchical clustering schemes. Psychometrika, 32, 241-254.

Kruskal, J. B. (1964a). Multidimensional scaling optimizing goodness of fit to a non-metric hypothesis. Psychometrika, 29, 1-27.

KRUSKAL, J. B. (1964b). Nonmetric multidimensional scaling: A numerical method. Psychometrika, 29, 115-129.

Kruskal, J. B., Young, F. W., Seery, J. B. (1977). How to use KYST $2 A$, a very flexible program to do multidimensional scaling and unfolding. Murray Hill, NJ: AT\&T Bell Laboratories.

Loomis, J. M. (1982). Analysis of tactile and visual confusion matrices. Perception \& Psychophysics, 31, 41-52.

OsGooD, G. (1988). Generalizing the Apple-Psych system. Behavior Research Methods, Instruments, \& Computers, 20, 155-157.

Posner, M. I. (1978). Chronometric explorations of mind. New York: Erlbaum.

Posner, M. I., Boies, S. J., Eichelman, W. H., a Taylor, R. L. (1969). Retention of visual and name codes of single letters. Journal of Experimental Psychology Monographs, 79(1, Pt. 2).

Pruzansky, S., Tversky, A., \& Carroll, J. D. (1982). Spatial versus tree representations of proximity data. Psychometrika, 47, 3-19.

SOKAL, R. R., Michener, C. D. (1958). A statistical method for evaluating systematic relationships. University of Kansas Science Bulletin, 38, 1409-1438.

Thorson, G., Hochraus, L., \& Stanners, R. F. (1976). Temporal changes in visual and acoustic codes in a letter-matching task. Perception \& Psychophysics, 19, 346-348.

TownsEnd, J. T. (1971). Theoretical analysis of an alphabetic confusion matrix. Perception \& Psychophysics, 9, 40-50.

van der Heijden, A. H. C., Malhas, M. S. M., \& van den Roovart, B. P. (1984). An empirical interletter confusion matrix for continuous-line capitals. Perception \& Psychophysics, 35, 85-88.

\section{NOTE}

1. A diskette formatted in Apple Pascal is available on request at no cost to anyone desiring facilitated access to the similarity ratings for modeling or other purposes. Send the request to David B. Boles, Department of Psychology, Rensselaer Polytechnic Institute, Troy, NY 12180. 
APPENDIX A

Mean Similarity Values, Each of Two Decimal Places

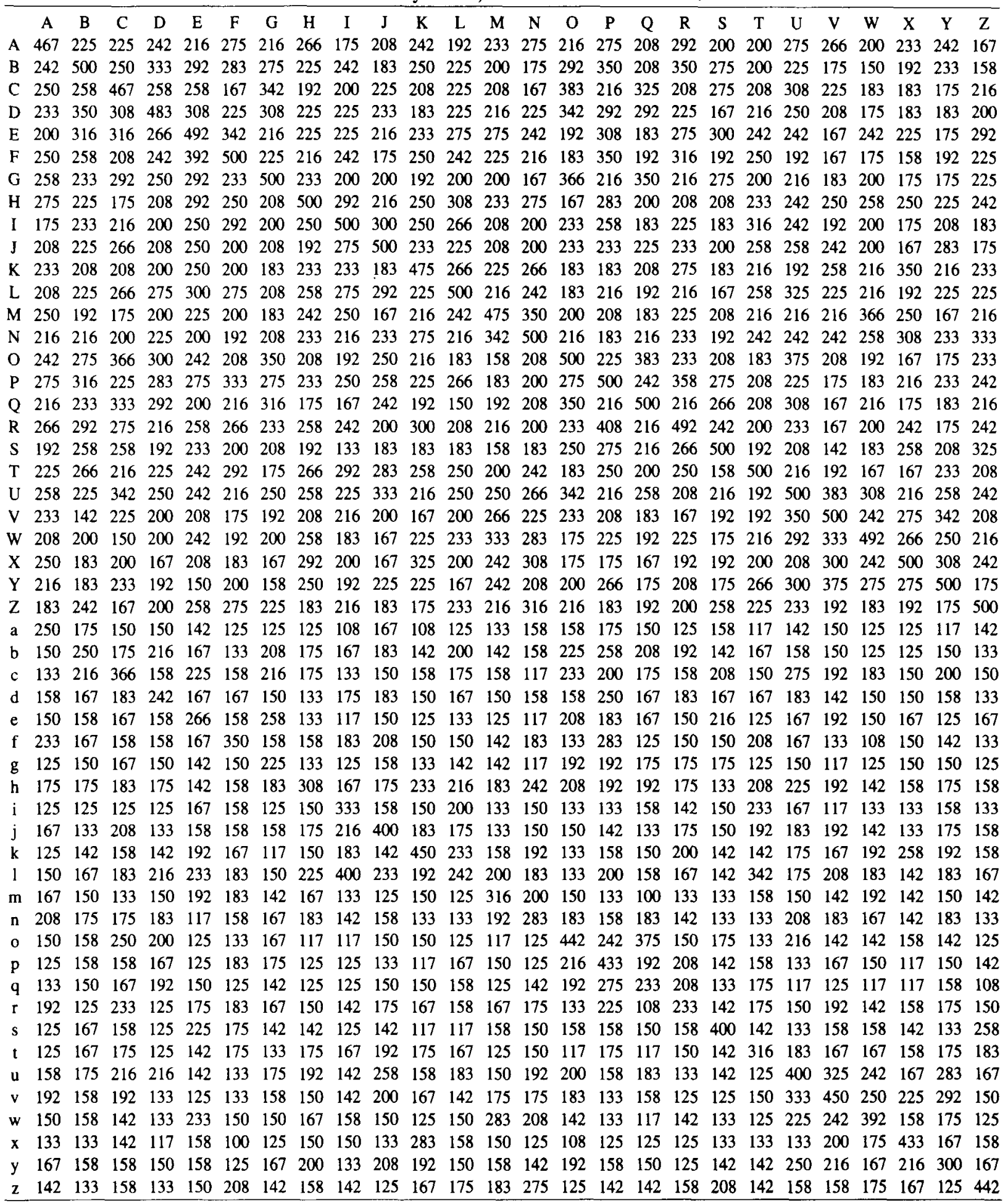

Note-left column $=$ first letter in pair. Top row $=$ second letter. 
Appendix A (Continued)

\begin{tabular}{|c|c|c|c|c|c|c|c|c|c|c|c|c|c|c|c|c|c|c|c|c|c|c|c|c|c|c|}
\hline$a$ & 0 &  &  & & 1 & $\mathbf{g}$ & h & 1 & J & $\mathbf{k}$ & & & 1 & 0 & & 1 & r & $\mathbf{S}$ & & 1 & & & & y & & \\
\hline & & 5 & 8 & & & 17 & & & & 150 & 158 & & & 150 & & & 167 & & & & 167 & & & & & \\
\hline & & 67 & 0 & & & 5 & & & & 2 & & & & 192 & & & & & & & 142 & & & 88 & & \\
\hline & & 88 & 88 & & & 42 & & & 0 & se & & & & 216 & & 67 & 216 &  & 9 & & 200 & & & 50 & & \\
\hline & & 42 & 42 & & & 58 & & & & 135 & 00 & & & 192 & & & 142 & & & & & & & & & \\
\hline & 57 & 08 & 17 & 50 & 2 & 42 & 50 & 42 & 3 & 18 & 83 & & & 133 & 67 & & 133 & 216 & 6 & 57 & 150 & & 150 & 50 & & \\
\hline & & 83 & 42 & & 366 & 25 & 08 & & & & 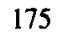 & & & 150 & & & & & & & & & & & & \\
\hline & & 66 & 200 & 25 & 0 & 16 & 42 & 50 & 50 & 117 & 33 & & & 192 & & , & 183 & 158 & 67 & 00 & 150 & & 2 & 50 & 3. & \\
\hline & & 50 & 75 & & & & & & & & 00 & & & - & & & & & & & & & & & & \\
\hline & & 42 & 67 & & 33 & & 200 & 2 & 57 & $?$ & 17 & & & 125 & & & 8 & 15 & & & 14 & & & 7 & & \\
\hline & & 75 & 15 & & 25 & 175 & 58 & & & 183 & 42 & & & 158 & & & 15 & 80 & & & 216 & & & 33 & 5 & \\
\hline & & 33 & 50 & & 33 & & 83 & & 42 & 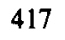 & 00 & & & 142 & & 2 & 192 & 133 & & & 19 & & 5 & 80 & 17 & \\
\hline & & 92 & 58 & & 0 & 33 & 92 & & 75 & & 66 & & & 125 & & & 1 & 13 & & & 17 & & & ) & 17 & \\
\hline & & 50 & 33 & & 3 & 24 & 00 & & 2 & & 83 & & & 13 & & & & & & & & & & & & \\
\hline & & 58 & 25 & & 17. & & 250 & & 15 & 225 & 08 & & & 125 & & & 15 & 150 & & & 17 & & 08 & 6 & 292 & \\
\hline & & 75 & 16 & & & & 175 & & & & & & & 4 & & & & & & & & & & & & \\
\hline & & 08 & 58 & & 258 & $n$ & 192 & & 33 & 15 & & & & 242 & & & 26 & 15 & & & 12 & & & 12 & 16 & \\
\hline & & 08 & 2 & & & & & & & & & & & & & & & & & & & & & & & \\
\hline & & 08 & 00 & & 2 & & 58 & & 67 & - & & & & 16 & & & 2 & 158 & & & 15 & & & 3 & 16 & 2 \\
\hline & & 25 & 50 & & & & & & & & & & & & & & & & & & & & & & & \\
\hline & & 33 & 75 & & 8 & & 75 & & 142 & & 92 & & & 150 & & & l & 14 & & & I & & & & 10 & \\
\hline & & 58 & 33 & & & & 16 & & & & & & & 2 & & & & 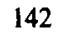 & & & & & & & 14 & \\
\hline & & 92 & 58 & & & & 2 & & & & 58 & & & 16 & & & & 1 & & & & & & & 150 & \\
\hline & & 33 & & & & & & & & & & & & 1 & & & & & & & & & & & 15 & \\
\hline & & 67 & 50 & & & & 12 & & 8 & & 25 & & & 1 & & & & 14 & & & & & & & & \\
\hline & & 16 & & & & & 88 & & & & & & & 1. & & & & 1 & & & 2 & & & & 12. & \\
\hline & & 8 & 33 & & & & 17 & & 42 & & & & & 133 & & & & 24 & & & & & & & & \\
\hline & & 33 & 33 & & & 42 & 83 & & 8 & & & & & 300 & & & & 2 & & & 2 & & & & 242 & \\
\hline & &  & 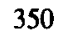 & & & 26 & 442 & & 16 & & & & & 28 & & & & 23 & & & 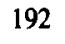 & & & & 15 & \\
\hline & & 83 & 42 & 10 & 42 & 25 & 58 & 58 & 216 & 15 & 33 & & & 342 & & 12 & 292 & 275 & 200 & 25 & 292 & & & 208 & 233 & \\
\hline & & 2.5 & 0 & & & &  & & & & & & & 26 & & & & & & & & & & & & \\
\hline & & 25 & 50 & & 88 & 30 & 83 & & 175 & & 42 & & & 325 & & & 3 & 283 & & & 192 & & & 58 & 25 & e \\
\hline & & 08 & & & & & & & & & & & & 1 & & & & & & & & & & & & \\
\hline & & 66 & 15 & & 0 & 50 & 16 & & 56 & & & & & 258 & & & 175 & 233 & & & 83 & & & & 158 & E \\
\hline & & & & & & & & & & & & & & 1 & & & & 1. & & & & & & & & \\
\hline & & 167 & 183 & & 192 & & 175 & & 358 & & 392 & & & 183 & & & 192 & 183 & & & 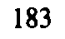 & & & & 175 & \\
\hline & & - & 8 & & & & 00 & & 5 & & & & & 19 & & & & 20 & & & & & & & 2 & \\
\hline & & 00 & 16 & & & & 25 & & 150 & & ? & & & 142 & & & 20 & 175 & & & 233 & & & & 18 & \\
\hline & & 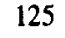 & 25 & & & . & 08 & & 208 & & 0 & & & 142 & & & & 175 & & & 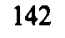 & & & & 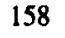 & 1 \\
\hline & & & & & & & & & & & & & & 2 & & & & I & & & & & & & . & \\
\hline & & & & & & & & & & & & & & & & & & & & & & & & & & \\
\hline & & 16 & 6 & & 30 & 23 & 8 & & 216 & 15 & 133 & & & 500 & & & 15 & 233 & & & 283 & & & & 158 & \\
\hline & & & & & & & & & 216 & & & & & 30 & & & 2 & 23 & & & 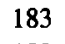 & & & & 2 & \\
\hline & & 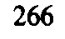 & & & 2 & & & & & & & & & 242 & & & & 183 & & & -7 & & & & 175 & \\
\hline & & & 2 & & & & & & 233 & & & & & 266 & & & 5 & 21 & & & 20 & & & & 20 & \\
\hline & & 92 & & & 88 & & 58 & & & & & & & 20 & & & 2 & 4 & & & 15 & & & & 3 & \\
\hline & & 08 & 225 & & 342 & 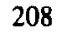 & 00 & 0 & 233 & & & & & 192 & & & 192 & 183 & & 92 & 233 & & & & 216 & \\
\hline & & & & & 18 & & & & 20 & & 10 & & & 29 & & & 21 & 200 & 13 & & 400 & & & & 22 & \\
\hline & & 92 & 75 & & 167 & & 22 & & 225 & & & & & 250 & & & 08 & 208 & 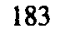 & & 500 & & & & 24 & \\
\hline & & 216 & & & 175 & 18 & & & 175 & & & & & 15 & & & 18 & 24 & & & 32 & & & & 25 & \\
\hline & & 25 &  & $x$ & 142 & 11 & 183 & 1 & 158 & 39 & 142 & 20 & & $16^{\circ}$ & & 20 & 20 & 17 & ( & 225 & 25 & & 0 & & 258 & \\
\hline & & 16 & 50 & & 175 & 36 & 50 & & 266 & 216 & & 21 & & 208 & & 275 & 225 & 216 & 19 & 58 & 350 & 58 & 233 & 500 & 192 & \\
\hline & & 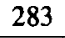 & & & 200 & . & 100 & & & & & & & 110 & & & & (5) & 100 & & 16 & & & 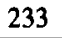 & & \\
\hline
\end{tabular}


APPENDIX B

Mean Generation and Veridical Similarity Values with Order Collapsed

\begin{tabular}{|c|c|c|c|c|c|c|c|c|c|c|c|c|c|c|c|c|c|c|c|c|c|c|c|c|c|c|}
\hline & $\mathbf{a}$ & b & c & $\mathrm{d}$ & e & f & g & h & $\mathrm{i}$ & $\mathrm{j}$ & k & 1 & $\mathrm{~m}$ & n & 0 & $\mathrm{p}$ & $q$ & $r$ & $\mathbf{s}$ & $t$ & $\mathbf{u}$ & $v$ & w & $x$ & $y$ & $z$ \\
\hline \multirow[t]{2}{*}{ A } & 484 & 240 & 248 & 232 & 272 & 232 & 261 & 227 & 179 & 187 & 191 & 179 & 233 & 240 & 253 & 259 & 222 & 259 & 227 & 194 & 252 & 212 & 195 & 214 & 210 & 210 \\
\hline & 237 & 152 & 154 & 148 & 152 & 166 & 133 & 171 & 123 & 148 & 126 & 141 & 152 & 172 & 163 & 159 & 152 & 163 & 159 & 144 & 150 & 159 & 142 & 123 & 140 & 145 \\
\hline \multirow[t]{2}{*}{ B } & & 500 & 261 & 355 & 286 & 225 & 275 & 278 & 198 & 190 & 244 & 221 & 183 & 208 & 290 & 342 & 290 & 278 & 233 & 232 & 221 & 174 & 163 & 174 & 221 & 183 \\
\hline & & 263 & 195 & 210 & 171 & 154 & 190 & 183 & 152 & 156 & 150 & 180 & 148 & 154 & 207 & 230 & 171 & 170 & 167 & 161 & 165 & 148 & 145 & 127 & 148 & 133 \\
\hline \multirow[t]{2}{*}{ C } & & & 475 & 259 & 300 & 182 & 290 & 214 & 185 & 233 & 193 & 188 & 202 & 257 & 353 & 237 & 292 & 265 & 275 & 208 & 323 & 259 & 190 & 193 & 221 & 225 \\
\hline & & & 413 & 210 & 210 & 162 & 198 & 171 & 133 & 183 & 143 & 173 & 143 & 158 & 244 & 181 & 179 & 204 & 181 & 147 & 244 & 194 & 145 & 145 & 181 & 152 \\
\hline \multirow[t]{2}{*}{$\mathrm{D}$} & & & & 492 & 259 & 216 & 302 & 263 & 198 & 214 & 206 & 235 & 195 & 210 & 307 & 302 & 330 & 240 & 192 & 228 & 240 & 195 & 171 & 166 & 210 & 170 \\
\hline & & & & 242 & 159 & 148 & 165 & 166 & 148 & 169 & 143 & 185 & 153 & 162 & 192 & 212 & 182 & 163 & 144 & 148 & 193 & 143 & 139 & 133 & 152 & 133 \\
\hline \multirow[t]{2}{*}{$\mathrm{E}$} & & & & & 488 & 290 & 261 & 230 & 195 & 203 & 203 & 218 & 225 & 223 & 271 & 280 & 208 & 280 & 277 & 208 & 255 & 190 & 208 & 206 & 175 & 273 \\
\hline & & & & & 259 & 167 & 192 & 141 & 140 & 147 & 159 & 174 & 159 & 133 & 171 & 173 & 150 & 157 & 210 & 144 & 152 & 154 & 175 & 152 & 144 & 163 \\
\hline \multirow[t]{2}{*}{$\mathrm{F}$} & & & & & & 500 & 227 & 216 & 229 & 225 & 194 & 257 & 198 & 195 & 170 & 263 & 194 & 271 & 193 & 296 & 190 & 182 & 179 & 158 & 188 & 208 \\
\hline & & & & & & 359 & 145 & 170 & 179 & 184 & 165 & 165 & 154 & 154 & 141 & 233 & 129 & 181 & 157 & 195 & 145 & 134 & 134 & 125 & 142 & 160 \\
\hline \multirow[t]{2}{*}{$\mathrm{G}$} & & & & & & & 500 & 207 & 194 & 240 & 188 & 184 & 206 & 191 & 303 & 284 & 388 & 216 & 233 & 195 & 225 & 188 & 188 & 173 & 271 & 200 \\
\hline & & & & & & & 221 & 154 & 129 & 160 & 125 & 139 & 145 & 145 & 180 & 185 & 169 & 175 & 173 & 135 & 165 & 137 & 134 & 129 & 159 & 131 \\
\hline \multirow[t]{2}{*}{ H } & & & & & & & & 500 & 228 & 208 & 286 & 242 & 233 & 298 & 191 & 257 & 205 & 286 & 177 & 227 & 282 & 233 & 216 & 218 & 249 & 195 \\
\hline & & & & & & & & 321 & 173 & 165 & 193 & 208 & 181 & 223 & 158 & 161 & 154 & 160 & 145 & 175 & 207 & 165 & 163 & 157 & 193 & 158 \\
\hline \multirow[t]{2}{*}{ I } & & & & & & & & & 484 & 321 & 208 & 326 & 212 & 205 & 202 & 223 & 163 & 216 & 173 & 270 & 218 & 191 & 183 & 182 & 192 & 192 \\
\hline & & & & & & & & & 313 & 175 & 163 & 294 & 139 & 157 & 133 & 154 & 139 & 143 & 139 & 201 & 140 & 135 & 145 & 134 & 137 & 135 \\
\hline $\mathrm{J}$ & & & & & & & & & & 500 & 185 & 249 & 169 & 214 & 223 & 240 & 207 & 232 & 191 & 271 & 273 & 230 & 177 & 169 & 50 & 179 \\
\hline & & & & & & & & & & 379 & 163 & 207 & 135 & 148 & 160 & 163 & 141 & 173 & 145 & 195 & 202 & 192 & 145 & 126 & 198 & 143 \\
\hline $\mathbf{K}$ & & & & & & & & & & & 488 & 228 & 198 & 206 & 175 & 199 & 182 & 237 & 168 & 223 & 195 & 214 & 198 & 359 & 226 & 197 \\
\hline & & & & & & & & & & & 434 & 221 & 166 & 177 & 139 & 135 & 150 & 201 & 131 & 152 & 171 & 180 & 169 & 273 & 175 & 161 \\
\hline $\mathrm{L}$ & & & & & & & & & & & & 500 & 192 & 190 & 160 & 198 & 175 & 179 & 163 & 277 & 229 & 183 & 190 & 175 & 175 & 190 \\
\hline & & & & & & & & & & & & 255 & 156 & 168 & 137 & 173 & 153 & 161 & 137 & 242 & 190 & 171 & 167 & 139 & 177 & 167 \\
\hline $\mathbf{M}$ & & & & & & & & & & & & & 488 & 361 & 202 & 191 & 178 & 253 & 195 & 200 & 232 & 216 & 342 & 232 & 203 & 218 \\
\hline & & & & & & & & & & & & & 334 & 205 & 135 & 137 & 117 & 165 & 143 & 145 & 165 & 167 & 248 & 152 & 150 & 161 \\
\hline $\mathrm{N}$ & & & & & & & & & & & & & & 500 & 230 & 204 & 216 & 261 & 195 & 214 & 294 & 240 & 263 & 242 & 225 & 273 \\
\hline & & & & & & & & & & & & & & 304 & 145 & 134 & 152 & 156 & 145 & 147 & 210 & 179 & 194 & 163 & 165 & 214 \\
\hline 0 & & & & & & & & & & & & & & & 500 & 284 & 309 & 231 & 223 & 179 & 336 & 244 & 171 & 165 & 195 & 195 \\
\hline & & & & & & & & & & & & & & & 434 & 223 & 271 & 157 & 165 & 137 & 230 & 169 & 139 & 139 & 159 & 134 \\
\hline $\mathrm{P}$ & & & & & & & & & & & & & & & & 500 & 318 & 338 & 261 & 216 & 212 & 187 & 202 & 177 & 248 & 208 \\
\hline & & & & & & & & & & & & & & & & 413 & 235 & 237 & 154 & 157 & 154 & 139 & 131 & 127 & 150 & 142 \\
\hline Q & & & & & & & & & & & & & & & & & 500 & 216 & 214 & 187 & 250 & 183 & 191 & 175 & 223 & 188 \\
\hline & & & & & & & & & & & & & & & & & 225 & 162 & 143 & 150 & 169 & 137 & 127 & 125 & 161 & 133 \\
\hline $\mathrm{R}$ & & & & & & & & & & & & & & & & & & 496 & 233 & 227 & 244 & 198 & 198 & 218 & 206 & 225 \\
\hline & & & & & & & & & & & & & & & & & & 233 & 163 & 163 & 152 & 156 & 142 & 150 & 154 & 161 \\
\hline $\mathbf{S}$ & & & & & & & & & & & & & & & & & & & 492 & 183 & 208 & 183 & 210 & 216 & 197 & 307 \\
\hline & & & & & & & & & & & & & & & & & & & 417 & 144 & 139 & 148 & 150 & 135 & 142 & 237 \\
\hline$T$ & & & & & & & & & & & & & & & & & & & & 484 & 198 & 200 & 183 & 183 & 221 & 208 \\
\hline & & & & & & & & & & & & & & & & & & & & 309 & 141 & 159 & 150 & 139 & 167 & 152 \\
\hline $\mathrm{U}$ & & & & & & & & & & & & & & & & & & & & & 471 & 384 & 325 & 214 & 329 & 233 \\
\hline & & & & & & & & & & & & & & & & & & & & & 409 & 338 & 255 & 163 & 266 & 155 \\
\hline V & & & & & & & & & & & & & & & & & & & & & & 500 & 302 & 280 & 349 & 205 \\
\hline & & & & & & & & & & & & & & & & & & & & & & 429 & 253 & 227 & 261 & 147 \\
\hline 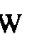 & & & & & & & & & & & & & & & & & & & & & & & 496 & 222 & 257 & 232 \\
\hline & & & & & & & & & & & & & & & & & & & & & & & 409 & 182 & 180 & 154 \\
\hline 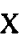 & & & & & & & & & & & & & & & & & & & & & & & & 500 & 248 & 233 \\
\hline & & & & & & & & & & & & & & & & & & & & & & & & 417 & 185 & 167 \\
\hline 1 & & & & & & & & & & & & & & & & & & & & & & & & & 500 & 194 \\
\hline & & & & & & & & & & & & & & & & & & & & & & & & & 309 & 137 \\
\hline 2 & & & & & & & & & & & & & & & & & & & & & & & & & & 500 \\
\hline & & & & & & & & & & & & & & & & & & & & & & & & & & 442 \\
\hline
\end{tabular}

Note-upper entry $=$ generation similarity. Lower entry $=$ veridical similarity . 